\title{
Universal behavior of scalar dissipation rate in confined porous media
}

\author{
Marco De Paoli $\odot,{ }^{1, *}$ Vlad Giurgiu, ${ }^{1, \dagger}$ Francesco Zonta, ${ }^{1, \ddagger}$ and Alfredo Soldati ${ }^{1,2, \S}$ \\ ${ }^{1}$ Institute of Fluid Mechanics and Heat Transfer, TU Wien, 1060 Vienna, Austria \\ ${ }^{2}$ Polytechnic Department, University of Udine, 33100 Udine, Italy
}

(Received 24 July 2019; published 21 October 2019)

\begin{abstract}
We study the problem of fluid mixing induced by gravity-driven Rayleigh-Taylor convective dissolution in porous media with top and bottom confinement. The competition between buoyancy and diffusion is measured by the Rayleigh-Darcy number, $\mathrm{Ra}_{0}$, the value of which controls the entire dynamics of the flow. In this work, mixing is quantified by the mean scalar dissipation rate, $\langle\chi\rangle$. Our results indicate that the time behavior of $\langle\chi\rangle$ is nontrivial and distinctly different from previous observations in Rayleigh-Bénard configuration. Analyzing a number of simulations for a broad range of $\mathrm{Ra}_{0}$, we clearly show that, in the convection-dominated regime, $\langle\chi\rangle$ exhibits a sublinear growth $\left(\langle\chi\rangle \sim t^{0.3}\right)$, which, in the limit of large $\mathrm{Ra}_{0}$, is very robust, does not seem to depend on $\mathrm{Ra}_{0}$, and can pave the way for new modeling perspectives.
\end{abstract}

DOI: 10.1103/PhysRevFluids.4.101501

In this paper, we focus on a porous medium saturated by two fluids with different specific weights. The investigated domain has top and bottom confinements, and the two fluids are initially in an unstable configuration which is prone to Rayleigh-Taylor (RT) instability [1] (i.e., in which the heavier fluid sits on top of the lighter fluid). This RT instability, which is driven here by buoyancy effects due to solute concentration-induced density differences, naturally evolves into flow convection. The convection problem is, however, complicated by non-negligible diffusion effects that tend to homogenize the flow and to smooth over the existing solute concentration gradients. The competition between buoyancy-induced convection and diffusion generates different evolution scenarios. Motivated by the important environmental and geophysical implications such as water contamination [2], solute transport in the context of petroleum maturation and migration [3], and carbon dioxide sequestration [4], we decided to focus on the specific configuration in which the porous domain has impermeable top and bottom boundaries.

The key dimensionless parameter that controls the time evolution of the flow is the RayleighDarcy number $\mathrm{Ra}_{0}$, which measures the importance of buoyancy compared to diffusion [5]. While at small $\mathrm{Ra}_{0}$ the problem is controlled by diffusion, at large $\mathrm{Ra}_{0}$ gravity influences the overall physical picture by amplifying initially small flow perturbations, which in turn evolve into convective fingers and plumes. Regardless of the value of the Rayleigh-Darcy number, diffusion drives the initial system evolution, until instabilities grow and become large enough to induce buoyancy-driven convective flows. At this stage, the two fluids are vigorously mixed by convection. Quantifying the amount of mixing in this physical situation is an extremely important aspect to characterize fluid transport in a porous medium. Following previous studies in the field [6,7], we use the scalar

\footnotetext{
*marco.de.paoli@tuwien.ac.at

†vlad.giurgiu@tuwien.ac.at

francesco.zonta@tuwien.ac.at

§alfredo.soldati@tuwien.ac.at
} 
dissipation rate $\chi$ as a mixing indicator. The normalized integral of $\chi$ over the entire domain (i.e., normalized by the horizontal extension of the domain), which we refer to as $\langle\chi\rangle$, gives a clear and robust measure of the amount of mixing [8].

The RT-like configuration has received relatively less attention compared to the classical Rayleigh-Bénard (RB) configuration [9,10]: However, it exhibits features that are important in a number of geophysical applications, including saline soils contamination [2], petroleum migration [3], salinity inversion [11,12], and carbon dioxide sequestration [4,13].

Also important is to characterize the time behavior of mixing, which we do analyzing $\langle\chi\rangle$ for a range of $\mathrm{Ra}_{0}$ that can be relevant for environmental applications. Our results indicate that $\langle\chi\rangle$ has a nontrivial time-dependent behavior and does not achieve a constant steady-state value, like in the RB configuration $[7,9,14]$. We also show that, in the limit of large $\mathrm{Ra}_{0}$, the behavior of $\langle\chi\rangle$ appears universal. Finally, we advance a further step in the direction of modeling by computing the instantaneous Fourier modes, as well as the corresponding mean wave number, of the scalar dissipation rate along the horizontal centerline of the domain. This computation provides a quantitative and repeatable indication about the time behavior of the mixing rate occurring at the center of the domain, a parameter which is of paramount importance in the development of simplified phenomenological mass and solute transfer models. We believe that these results can open new perspectives for future modeling and parametrization of flow convection and mixing inside porous media.

We consider the two-dimensional (2D) flow of a binary mixture inside a porous layer characterized by uniform permeability $k$ and porosity $\phi$ and with dimensions $L^{*}$ and $H^{*}$ along the horizontal $\left(x^{*}\right)$ and vertical $\left(z^{*}\right)$ directions, respectively. The two fluids that compose the binary mixture have constant diffusivity $D$ and viscosity $\mu$ and are perfectly miscible but have different density that indeed does depend on the local solute concentration. The flow is initialized such that the upper half of the domain is filled with a heavier fluid (characterized by a solute concentration $C^{*}=C_{s}^{*}$, with $C_{s}^{*}$ the saturation value), while the lower half of the domain is filled with a lighter fluid (characterized by a solute concentration $C^{*}=0$ ). The initial density difference between the two fluids, $\Delta \rho^{*}=\rho^{*}\left(C_{s}^{*}\right)-\rho^{*}(0)$, induces the vertical buoyancy force (aligned with the acceleration due to gravity, $g$ ) that ultimately drives the fluid motion. When made dimensionless using the velocity scale $W^{*}=g k \Delta \rho^{*} / \mu$, the length scale $\ell^{*}=\phi D / W^{*}$, the timescale $\phi \ell^{*} / W^{*}$, and the concentration $C_{s}^{*}[14,15]$, the flow field $(u, w)$ is described by the Darcy law written under the Oberbeck-Boussinesq approximation [16]:

$$
u=-\frac{\partial P}{\partial x}, \quad w=-\frac{\partial P}{\partial z}-C, \quad \frac{\partial u}{\partial x}+\frac{\partial w}{\partial z}=0,
$$

where $P=p+z\left[\rho^{*}\left(C_{s}^{*}\right) / \Delta \rho^{*}-1\right]$ is the reduced pressure. Note that the superscript $*$, which is used to indicate dimensional variables, is dropped to refer to dimensionless variables. In the absence of dispersion effects, solute transport is governed by the following advection-diffusion equation:

$$
\frac{\partial C}{\partial t}+u \frac{\partial C}{\partial x}+w \frac{\partial C}{\partial z}=\frac{\partial^{2} C}{\partial x^{2}}+\frac{\partial^{2} C}{\partial z^{2}} .
$$

Using the proposed reference variables, the only governing parameter of the flow is the RayleighDarcy number, $\mathrm{Ra}_{0}=H^{*} / \ell^{*}=H$, which corresponds to the dimensionless height of the porous slab [15]. Impermeable boundary conditions are applied at the top and bottom sides $\left(w=0, \partial_{z} C=0\right.$ at $z=0$, and $z=\mathrm{Ra}_{0}$ ), whereas periodic boundary conditions are applied at the left and right sides $(x=0$ and $x=L)$. To initialize the flow, the solute concentration is $C\left(x, z>\mathrm{Ra}_{0} / 2, t=0\right)=1$ in the upper half of the domain and $C\left(x, z<\mathrm{Ra}_{0} / 2, t=0\right)=0$ in the lower half of the domain. A small random perturbation of the concentration field $\left(\varepsilon\right.$, made dimensionless by $\left.C_{s}^{*}\right)$ is applied along the interface of separation between the upper and the lower layers to trigger the initial flow instability (further details on the initial condition are given in the Supplemental Material [17]). A sketch of the computational domain is shown in Fig. 1, together with a contour map of the concentration field at a given time instant $\tilde{t}$ and the indication of the boundary conditions. 


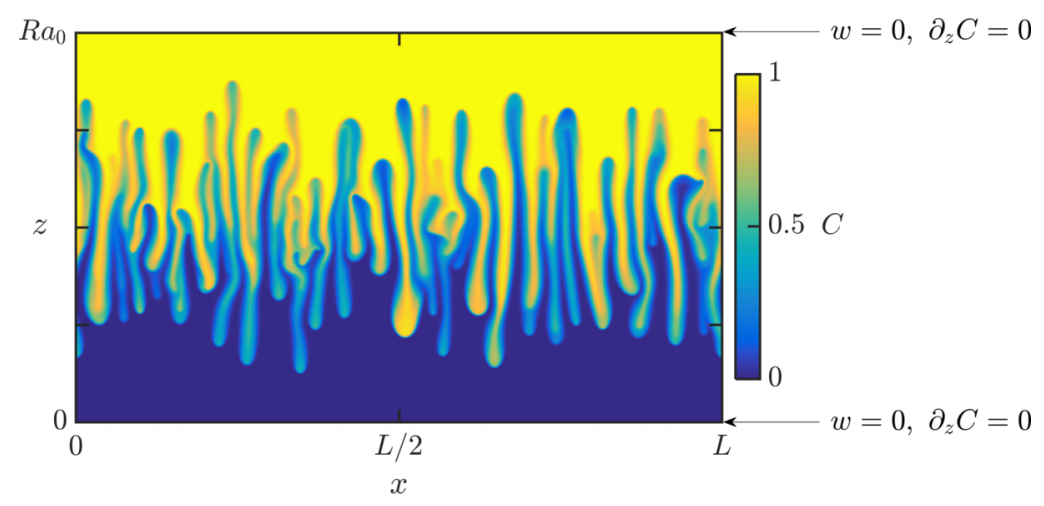

FIG. 1. Sketch of the computational domain with explicit indication of the boundary conditions. The contour map of the solute concentration $C(x, z, \tilde{t})$ for $\mathrm{Ra}_{0}=12023$ at $\tilde{t} \approx 2 \times 10^{4}$ is also shown. See the file Movie S1.mov in the Supplemental Material [17] for the time-dependent evolution of $C(x, z, t)$.

We consider a wide range of Rayleigh-Darcy numbers from $\mathrm{Ra}_{0}=955$ up to $\mathrm{Ra}_{0}=19553$, and, to avoid any possible influence of the domain width on the evolution of the system, we set $L=19953$ and therefore an aspect ratio $L / H \geqslant 1$ for all the simulations [18]. Equations (1) and (2) have been solved by a Fourier-Chebyshev pseudospectral method. Since the domain width $(L)$ is fixed for all the simulations, we keep the resolution constant (2048) in the horizontal direction, and we increase it with $\mathrm{Ra}_{0}$ in the vertical direction (up to 1025 collocation points). For further details on the numerical scheme we refer the reader to Refs. [14,19,20].

Usually, the degree of mixing is evaluated by examining the time behavior of the concentration variance $\sigma_{c}=\left\langle C^{2}\right\rangle-\langle C\rangle^{2}[6,21]$. Angular brackets $\langle\cdot\rangle$ indicate the integration of a quantity over the entire domain, normalized by the horizontal extension of the domain, $L^{*}$. The quantity $\sigma_{c}$ is maximum when the two fluids are segregated, while it drops down to $\sigma_{c}=0$ when the two fluids are perfectly mixed. Following Hidalgo et al. [7], multiplying Eq. (2) by $C$ and integrating over the entire domain (see also the Supplemental Material [17]), we can compute the time behavior of $\sigma_{c}$ as

$$
\frac{\partial \sigma_{c}}{\partial t}=\frac{\partial\left\langle C^{2}\right\rangle}{\partial t}=-2\left\langle|\nabla C|^{2}\right\rangle
$$

The quantity $|\nabla C|^{2}$ is called scalar dissipation rate [8,21] and is indicated with $\chi(x, z, t)$. The mean scalar dissipation rate $\langle\chi\rangle$ is a widely used mixing indicator since it is not influenced by ad hoc defined threshold coefficients, and it is proven very robust [7]. An in-depth discussion on $\langle\chi\rangle$ as a mixing indicator, and a vis-à-vis comparison of the behavior of $\langle\chi\rangle$ in different geophysically relevant situations (including RB and RT configuration) is provided in the Supplemental Material [17].

The evolution in time of $\langle\chi\rangle$ obtained by present numerical simulations is shown in Fig. 2(a) for $\mathrm{Ra}_{0}=12023$, a case for which the different aspects of the mixing process we wish to discuss are clearly visible. Three snapshots with the spatial distribution of $\chi$ at three different time instants are also shown in Figs. 2(b)-2(d). The analysis of Fig. 2 provides a comprehensive picture of the entire dynamics of dissolution-driven convection in the RT configuration and offers some interesting hints as to the corresponding amount of mixing. At the beginning, for $t<2 \times 10^{3}$, solute transport is purely controlled by diffusion. During this stage, the fluid velocity is negligible, and the thickness of the mixing region between the two fluid layers grows in time as $t^{1 / 2}[15,22]$. Accordingly, $\langle\chi\rangle$ evolves as $\langle\chi\rangle=t^{-1 / 2} / \sqrt{8 \pi}$ (see the Supplemental Material [17] for further details), confirming previous predictions [4]. We wish to remark here that the magnitude of the initial perturbation $O(\varepsilon)$ plays a role in the initial development of the flow: the larger the magnitude of $\varepsilon$, the sooner the onset of convection (we refer the reader to the Supplemental Material [17] for a deeper discussion of this aspect). The diffusion layer builds up in time until it reaches a critical thickness, which is large 


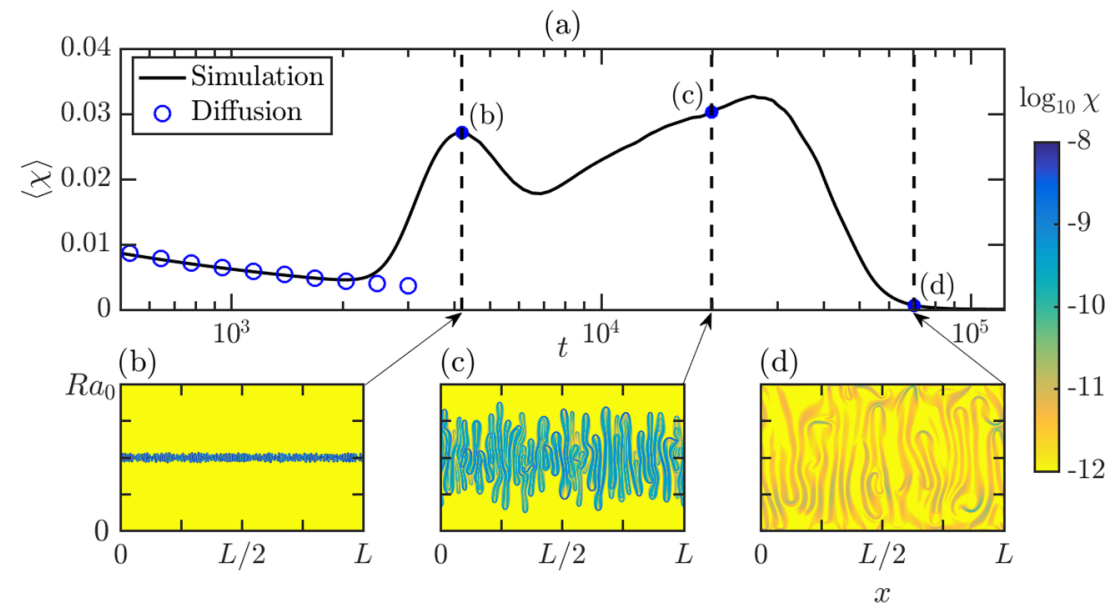

FIG. 2. Time evolution of the RT system characterized by $\mathrm{Ra}_{0}=12023$. (a) Mean scalar dissipation rate $\langle\chi\rangle$ (solid line) and diffusive analytical solution (open circles). The flow structure, rendered by contour maps of the scalar dissipation rate $\chi(x, z, t)$, is also explicitly given at three specific time instants: (b) emerging of fingering structures, with a local maximum of $\langle\chi\rangle$, (c) convection-dominated regime, and (d) shutdown of convection. See the file Movie S2.mov in the Supplemental Material [17] for the complete time-dependent evolution of $\chi(x, z, t)$.

enough to develop a buoyancy-induced instability that ultimately leads to the formation of fingers. This happens at $t \approx 2 \times 10^{3}$. Fingers, by stretching and deforming the interface between the two fluid layers, induce an increase of the mean scalar dissipation rate (and therefore of mixing). The interplay between the interface stretching process driven by fingers (which reduces the interface thickness and sharpens the concentration gradients) and the diffusion process (which tends to smooth out concentration gradients) produces a nontrivial behavior of $\langle\chi\rangle$ in time: $\langle\chi\rangle$ develops an initial crest at $t \approx 4 \times 10^{3}$ [Fig. 2(b)], due to a fast growth of the fingers and to a corresponding fingers-induced interface stretching, followed by a dip at $t \approx 7 \times 10^{3}$, due to a renewed influence of diffusion and of the corresponding merging of fingers. For $t>7 \times 10^{3}$, small fingers become larger plumes that move predominantly in the vertical direction [Fig. 2(c)] during a stage which is marked by plume elongation. After this stage $\left(t>2 \times 10^{4}\right)$, large plumes impinge on the wall, and the flow enters the convective shutdown stage characterized by a sharp drop of $\langle\chi\rangle$ [Fig. 2(d)]. During the shutdown stage, the confinement imposed by the boundaries progressively hinders convection and favors diffusion. When a stable density profile is established, i.e., when the light fluid sits on top of the heavy fluid, convection completely stops and diffusion acts, mixing the flow towards a final, perfectly homogenous solution characterized by uniform concentration (no gradients of solute concentration inside the flow).

The time behavior of the mean scalar dissipation rate $\langle\chi\rangle$ for all the values of $\mathrm{Ra}_{0}$ considered in this study is shown in Fig. 3(a). It is interesting to note that, in the limit of large $\mathrm{Ra}_{0}$, the evolution of $\langle\chi\rangle$ appears almost universal and only slightly influenced by $\mathrm{Ra}_{0}$ itself (with $\mathrm{Ra}_{0}$ influencing the time at which the shutdown stage starts). As already discussed before (see Fig. 2 and comments therein), during the diffusion stage $\left(t<2 \times 10^{3}\right)$ all curves collapse onto the theoretical prediction $\langle\chi\rangle=t^{-1 / 2} / \sqrt{8 \pi}$ [symbols in Fig. 3(a)]. Shortly after the diffusion stage, there is an intermediate stage (characterized by the competition between diffusion and convection [22]) during which the behavior of $\langle\chi\rangle$, although universal, is rather complex and cannot be easily parameterized. Later, during the stage dominated by convection $\left(t>7 \times 10^{3}\right)$, our results indicate that the mean scalar dissipation rate increases in time as $\langle\chi\rangle \sim t^{0.3}$, until the flow enters the shutdown stage. It is reasonable to argue that $\langle\chi\rangle \sim t^{0.3}$ is a truly universal asymptotic law emerging for $\operatorname{Ra}_{0} \rightarrow \infty$ (i.e., negligible diffusion). 

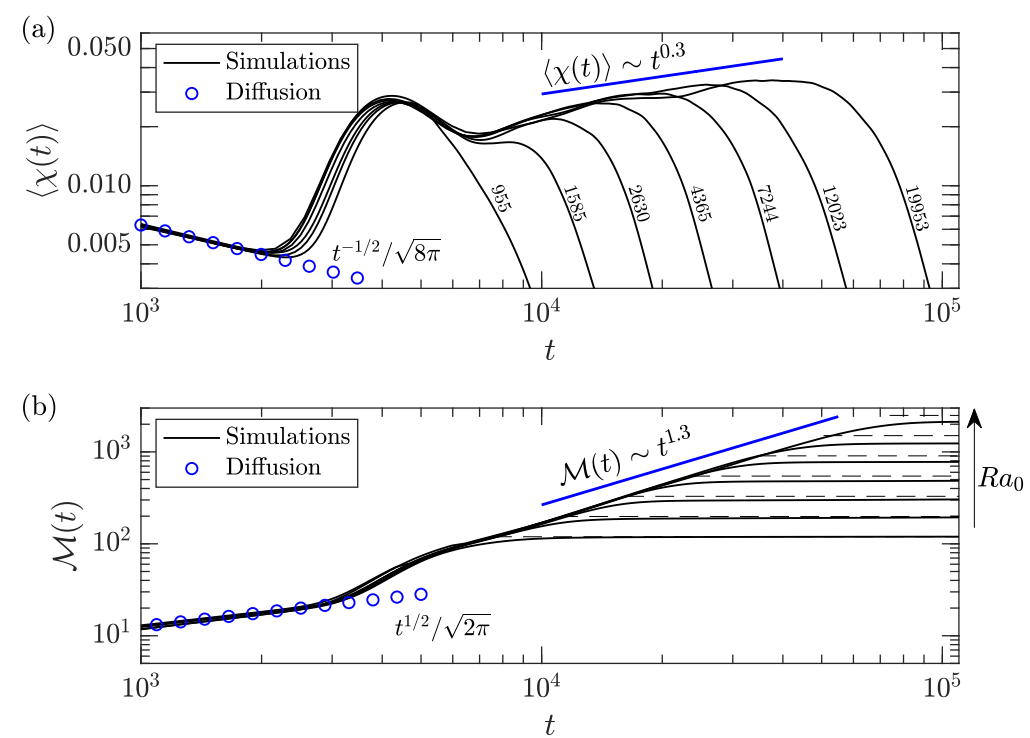

FIG. 3. Time evolution of the mean scalar dissipation rate $\langle\chi\rangle$ (a) and of the integral quantity $\mathcal{M}(t)$ (b) for different $\mathrm{Ra}_{0}$. The specific value of $\mathrm{Ra}_{0}$ is given in panel (a) close to each curve, while the amplitude of the initial perturbation is $O(\varepsilon)=10^{-5}$. The analytical evolution during the diffusive stage $\left(\langle\chi\rangle=t^{-1 / 2} / \sqrt{8 \pi}\right.$ and $\left.\mathcal{M}=t^{1 / 2} / \sqrt{2 \pi}\right)$ is also reported.

The universal behavior of mixing in the RT configuration appears even more clearly when inferred from the behavior of the mean scalar dissipation rate time integral, $\mathcal{M}(t)=\int_{0}^{t}\left\langle\chi\left(t^{\prime}\right)\right\rangle d t^{\prime}$, a quantity that bears interesting information about the history of the system. Our results, which are presented in Fig. 3(b) for the different $\mathrm{Ra}_{0}$ considered here, indicate fairly well the different scaling laws $\mathcal{M}(t) \sim t^{1 / 2}$ and $\mathcal{M}(t) \sim t^{1.3}$, which characterize the regimes dominated by diffusion and by convection, respectively [23]. Interestingly, $\mathcal{M}(t)$ has an asymptotic limit that can be predicted upon integration of Eq. (3) from $t=0$ to $t \rightarrow \infty$, i.e., upon evaluation of $\mathcal{M}_{\infty}=\left[\left\langle C^{2}(0)\right\rangle-\left\langle C^{2}(t \rightarrow \infty)\right\rangle\right] / 2$. From the initial condition, $C\left(x, z>\operatorname{Ra}_{0} / 2, t=0\right)=1$ and $C\left(x, z<\mathrm{Ra}_{0} / 2, t=0\right)=0$, we get $\left\langle C^{2}(0)\right\rangle=\mathrm{Ra}_{0} / 2$. For the final, and perfectly mixed condition $C(x, z, t \rightarrow \infty)=1 / 2$, we get $\left\langle C^{2}(\infty)\right\rangle=\mathrm{Ra}_{0} / 4$. This reasoning finally gives $\mathcal{M}(t \rightarrow \infty)=$ $\mathcal{M}_{\infty}=\mathrm{Ra}_{0} / 8$. Such prediction, which is explicitly shown for the different $\mathrm{Ra}_{0}$ by the dashed lines in Fig. 3(b), represents the asymptotic limit of the evolution of the system.

Finally, and to prepare further ground to develop currently available models of solute transport based on plume dynamics to the present flow configuration, we estimate the evolution in time of the flow structures along the horizontal centerline of the porous domain. We propose to use the Fourier decomposition of the scalar dissipation rate $\chi$ to extract the dominant flow features and to provide a clearer estimate of mixing evolution. This approach is based on the observation that the evolution of mixing between two species is determined by the behavior of two key quantities: the amount of interface separating the two species and the magnitude of the concentration gradient at the interface. Remarkably, $\chi$ contains information both on structure and extension of the interface and on the concentration gradient. We compute the power spectrum of $\chi$, and we evaluate the mean wave number as [24-26]

$$
\widehat{k}(t)=\frac{\int k_{n}\left|A_{n}\right|^{2} d k_{n}}{\int\left|A_{n}\right|^{2} d k_{n}} \quad \text { at } \quad z=\mathrm{Ra}_{0} / 2,
$$

with $k_{n}$ the wave number and $\left|A_{n}\right|^{2}$ the squared amplitude of the $n$th basic harmonic function obtained from the Fourier decomposition of $\chi$ along the domain centerline, $z=\operatorname{Ra}_{0} / 2$. Results 


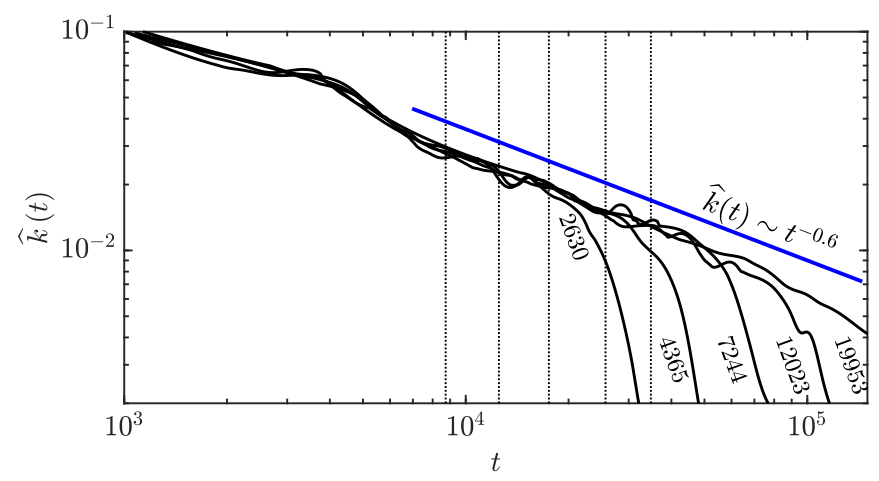

FIG. 4. Time evolution of the mean wave number $\widehat{k}(t)$, which is an estimate for the number of plumes in the interior part of the domain. The behavior is independent from $\mathrm{Ra}_{0}$ in the convective regime. See the file Movie S3.mov in the Supplemental Material [17] for the time-dependent evolution of the power spectrum during the entire mixing process.

are shown in Fig. 4 for $2630 \leqslant \mathrm{Ra}_{0} \leqslant 19953$. Note that the vertical dashed lines indicate the time at which plumes reach the boundary, $t_{i}$, for the different values of $\mathrm{Ra}_{0}$, therefore marking the beginning of the shutdown phase. Our calculation suggests that, during the stage of convective mixing $\left(t>7 \times 10^{3}\right)$, the mean wave number $\widehat{k}(t)=2 \pi n_{p} /(2 L)$ evolves in time as $\widehat{k} \sim t^{-0.6}$, where $n_{p}$ is the number of plumes (the coefficient 2 at the denominator is due to the fact that $\chi$ is maximum at the plume interface, i.e., twice per plume). This scaling extends far beyond the time instant at which plumes impinge on the boundaries and appears robust in the limit of large $\mathrm{Ra}_{0}$. At this stage of research, it seems hard to derive a physically based correspondence between the scaling of $\widehat{k}$ and that of $\langle\chi\rangle$.

To summarize, in this paper we have examined the behavior of fluid mixing for RT convective dissolution in confined porous media. More precisely, we have analyzed the time evolution of mixing by examining the mean scalar dissipation $\langle\chi\rangle$. Observing the time behavior of $\langle\chi\rangle$, it was possible to confirm that the entire dynamics of the RT convective dissolution is composed of three stages [15], each characterized by a different physical phenomenology. These stages are classified by the prevailing transport mechanism, which is initially diffusion, then convection, and finally diffusion again. Correspondingly, mixing behavior is profoundly different from stage to stage, and this is reflected by the time behavior of $\langle\chi\rangle$.

In the initial stage, diffusion dominates over buoyancy and, as in all alike diffusion-dominated processes, mixing decreases with $\langle\chi\rangle \sim t^{-1 / 2}$. In the second stage, convection prevails and mixing is promoted. Vigorous phenomena occurring in this stage control the largest proportion of mixing in the domain, and we have observed a corresponding increase of $\langle\chi\rangle$, which goes as $\sim t^{0.3}$. It is important to underline here that this time growth has a character distinctly different from analogous phenomena in similar flows, such as RB convection $[5,9,14]$. The final stage, which takes over once the vigorous convection starts decreasing its influence, is called shutdown [4], with slower diffusion phenomena again prevailing. The reported time scaling of $\langle\chi\rangle$ observed during the convective stage can be of paramount practical importance: It can be a very useful guideline to derive simple models. For example, it can give a quick estimate of the amount of solute mixed within the convective stage in geophysical applications [2,3]. There is some evidence that the present flow phenomenology and the corresponding scaling laws, obtained by 2D simulations, are consistent with those obtained by three-dimensional (3D) simulations [24]. However, future computations in 3D domains, possibly including additional effects like fluid miscibility and/or chemical reactions [25,27,28], are foreseen to improve current knowledge and physical modeling of solute transport in confined porous media. 
We acknowledge the Vienna Scientific Cluster (VSC) for generous allowance of computational resources.

[1] G. Boffetta and A. Mazzino, Incompressible Rayleigh-Taylor turbulence, Annu. Rev. Fluid Mech. 49, 119 (2017).

[2] W. H. Van Der Molen and H. C. Van Ommen, Transport of solutes in soils and aquifers, J. Hydrol. 100, 433 (1988).

[3] C. T. Simmons, T. R. Fenstemaker, and J. M. Sharp Jr., Variable-density groundwater flow and solute transport in heterogeneous porous media: Approaches, resolutions and future challenges, J. Contam. Hydrol. 52, 245 (2001).

[4] J. J. Hidalgo, M. Dentz, Y. Cabeza, and J. Carrera, Dissolution patterns and mixing dynamics in unstable reactive flow, Geophys. Res. Lett. 42, 6357 (2015).

[5] A. C. Slim, Solutal-convection regimes in a two-dimensional porous medium, J. Fluid Mech. 741, 461 (2014).

[6] T. Le Borgne, M. Dentz, D. Bolster, J. Carrera, J. R. De Dreuzy, and P. Davy, Non-Fickian mixing: Temporal evolution of the scalar dissipation rate in heterogeneous porous media, Adv. Water Resour. 33, 1468 (2010).

[7] J. J. Hidalgo, J. Fe, L. Cueto-Felgueroso, and R. Juanes, Scaling of Convective Mixing in Porous Media, Phys. Rev. Lett. 109, 264503 (2012).

[8] E. Villermaux, Mixing by porous media, Compt. Rend. Mécan. 340, 933 (2012).

[9] D. R. Hewitt, J. A. Neufeld, and J. R. Lister, Convective shutdown in a porous medium at high Rayleigh number, J. Fluid Mech. 719, 551 (2013).

[10] H. E. Huppert and J. A. Neufeld, The fluid mechanics of carbon dioxide sequestration, Annu. Rev. Fluid Mech. 46, 255 (2014).

[11] L. S. Land, Evidence for vertical movement of fluids, Gulf Coast sedimentary basin, Geophys. Res. Lett. 18, 919 (1991).

[12] R. A. Morton and L. S. Land, Regional variations in formation water chemistry, Frio formation (Oligocene), Texas Gulf Coast, AAPG Bull. 71, 191 (1987).

[13] G. Sardina, L. Brandt, G. Boffetta, and A. Mazzino, Buoyancy-Driven Flow through a Bed of Solid Particles Produces a New Form of Rayleigh-Taylor Turbulence, Phys. Rev. Lett. 121, 224501 (2018).

[14] M. De Paoli, F. Zonta, and A. Soldati, Dissolution in anisotropic porous media: Modelling convection regimes from onset to shutdown, Phys. Fluids 29, 026601 (2017).

[15] M. De Paoli, F. Zonta, and A. Soldati, Rayleigh-Taylor convective dissolution in confined porous media, Phys. Rev. Fluids 4, 023502 (2019).

[16] A. J. Landman and R. J. Schotting, Heat and brine transport in porous media: The Oberbeck-Boussinesq approximation revisited, Trans. Porous Med. 70, 355 (2007).

[17] See Supplemental Material at http://link.aps.org/supplemental/10.1103/PhysRevFluids.4.101501 for further details and the movies referenced in the figure captions.

[18] B. Wen, G. P. Chini, N. Dianati, and C. R. Doering, Computational approaches to aspect-ratio-dependent upper bounds and heat flux in porous medium convection, Phys. Lett. A 377, 2931 (2013).

[19] M. De Paoli, F. Zonta, and A. Soldati, Influence of anisotropic permeability on convection in porous media: Implications for geological $\mathrm{CO}_{2}$ sequestration, Phys. Fluids 28, 056601 (2016).

[20] F. Zonta and A. Soldati, Effect of temperature dependent fluid properties on heat transfer in turbulent mixed convection, J. Heat Transfer 136, 022501 (2014).

[21] E. Villermaux, Mixing versus stirring, Annu. Rev. Fluid Mech. 51, 245 (2019).

[22] S. S. Gopalakrishnan, J. Carballido-Landeira, A. De Wit, and B. Knaepen, Relative role of convective and diffusive mixing in the miscible Rayleigh-Taylor instability in porous media, Phys. Rev. Fluids 2, 012501 (2017). 
[23] The value of the exponent $\alpha=1.3$ (or, more precisely, $\alpha=1.298 \pm 0.004$ ) that appears in the scaling law $\mathcal{M}(t) \sim t^{\alpha}$ is determined via exponential fitting of the numerical results for $\mathcal{M}(t)$ in the convective regime. The scaling law of the mean scalar dissipation rate, $\langle\chi(t)\rangle \sim t^{0.3}$, is in turn obtained considering that $\langle\chi(t)\rangle=\partial_{t} \mathcal{M}(t)$.

[24] D. R. Hewitt, J. A. Neufeld, and J. R. Lister, High Rayleigh number convection in a three-dimensional porous medium, J. Fluid Mech. 748, 879 (2014).

[25] A. De Wit, Miscible density fingering of chemical fronts in porous media: Nonlinear simulations, Phys. Fluids 16, 163 (2004).

[26] A. Riaz, M. Hesse, H. A. Tchelepi, and F. M. Orr, Onset of convection in a gravitationally unstable diffusive boundary layer in porous media, J. Fluid Mech. 548, 87 (2006).

[27] L. Cueto-Felgueroso and R. Juanes, A phase-field model of two-phase Hele-Shaw flow, J. Fluid Mech. 758, 522 (2014).

[28] X. Fu, L. Cueto-Felgueroso, D. Bolster, and R. Juanes, Rock dissolution patterns and geochemical shutdown of $\mathrm{CO}_{2}$-brine-carbonate reactions during convective mixing in porous media, J. Fluid Mech. 764, 296 (2015). 